\title{
The Predictive Performance of the STOP-Bang Questionnaire in Obstructive Sleep Apnea Screening of Obese Population at Sleep Clinical Setting
}

Haluk Mergen ${ }^{1}$, Burak Altındağ ${ }^{1}$, Zeynep Zeren Uçar ${ }^{2}$, Işıl Karasu Kılıçaslan ${ }^{2}$

1. Family Medicine, University of Health Sciences, Izmir Tepecik Health Practice \& Research Center, Izmir, TUR 2. Sleep Medicine, University of Health Sciences, Izmir Dr. Suat Seren Health Practice \& Research Center, Izmir, TUR

Corresponding author: Haluk Mergen, haluk.mergen@gmail.com

\section{Abstract}

\section{Background}

The prevalence of obstructive sleep apnea (OSA) is high in the obese population. In this study, it was aimed to fulfill the STOP-Bang questionnaire which is a concise and easy-to-use questionnaire for OSA screening in obese patients.

\section{Materials \& methods}

This is a retrospective study where the patients, who planned polysomnography, were referred to sleep clinic. Patients were screened for OSA by the STOP-Bang questionnaire. Laboratory polysomnography was performed in 275 patients. Patients with $\mathrm{BMI} \geqslant 30$ were taken into study. The screening test was evaluated by three different risk analysis such as, a STOP score, a STOP-Bang score and a modified STOP-Bang score. The predictive parameters (sensitivity, specificity, and positive and negative predictive values) for alternative scoring models in obese patients were analyzed.

\section{Results}

In 217 obese patients, a STOP score cutoff of 3 and a STOP-Bang score cutoff of 4 provides a better balance of sensitivity and specificity for all OSA (apnea-hypopnea index [AHI] $\geqslant 5$ ). The STOP questionnaire revealed a sensitivity of $87.9 \%$ and a positive predictive value of $99.5 \%$ for patients with all OSA (p: 0.005). The STOPBang scoring model revealed a sensitivity of $95.3 \%$ and a positive predictive value of $99.5 \%$ for patients with all OSA ( $p<0.001$ ). The modified STOP-Bang scoring revealed a sensitivity of $95.8 \%$ and a positive predictive value of $99.5 \%$ for patients with all OSA ( $\mathrm{p}<0.001)$. The area under the curve of the STOP-Bang for identifying mild, moderate and severe OSA was $0.581,0.652$ and 0.675 , respectively. Whereas according to the STOP-Bang, all morbid obese patients (obesity class III, n: 47) were at high risk of OSA.

Received 10/10/2019

Review began 12/11/2019 Review ended 12/25/2019 Published 12/29/2019

\section{() Copyright 2019}

Mergen et al. This is an open access article distributed under the terms of the Creative Commons Attribution License CC-BY 3.0., which permits unrestricted use, distribution, and reproduction in any medium, provided the original author and source are credited.

\section{Conclusion}

This study suggests that the STOP-Bang questionnaire for obstructive sleep apnea screening in obese patients is a high sensitivity and appropriate screening test.

Categories: Family/General Practice, Pulmonology, Healthcare Technology

Keywords: : obstructive sleep apnea, obesity, stop-bang questionnaires, sleep apnea screening

\section{Introduction}

The prevalence of obstructive sleep apnea (OSA) was found to range from $3.1 \%$ to $7.5 \%$ in males and from $2.1 \%$ to $4.5 \%$ in females in studies conducted in different societies [1-6]. The most common symptoms of OSA are snoring, probable apnea, excessive daytime sleepiness, waking up with choking sensation, insomnia [7-9]. Although many symptoms occur during the day and night, most of the patients are not aware of their illness [10].

OSA is a treatable disease. However, overnight polysomnography (PSG), which is the gold standard for the diagnosis of OSA, is a difficult diagnostic method to reach [11]. Polysomnography is not available in many centers and the appointment times are long because it is a time consuming process [12]. Similar to many diseases, OSA has also developed questionnaires based on clinical and laboratory findings. In the questionnaires, sleep quality, symptoms of sleep disturbance, risk factors of sleep disturbance and possible complications related to sleep problems are questioned.

According to the World Health Organization in 2016, more than 1.9 billion adults, 18 years and older, were overweight. Of these, over 650 million were obese. The prevalence of OSA is high in the obese population. 
OSA risk was increased 8-12 times in cases with BMI > 29 [13]. In this study, it was aimed to apply the STOPBang questionnaire among obese patients which is not most studied.

\section{Materials And Methods}

The study was conducted after the approval of the Ministry of Health Sciences, Izmir Tepecik Education and Research Hospital Ethics Committee with the decision of 1 date and number 1. Between January 2017 and December 2017, adult patients, who applied to Sleep Disorders Clinic, Dr. Suat Seren Chest Diseases and Surgery Training and Research Hospital, because of obstructive sleep apnoea syndrome (OSAS) doubt and performed PSG, were included in the study. Patients with BMI 30-34.9 were classified as class 1 obese and patients with BMI 35-39.9 were classified as class 2 obese and patients with BMI $\geqslant 40$ were classified as class 3 (morbid obese). Patients with a BMI < 30 were not included in the study. Before the STOP-Bang questionnaire was filled, patients were informed and their consent was obtained.

\section{STOP-Bang survey}

The STOP-Bang questionnaire consists of eight questions. These questions are answered as "yes" or "no". The STOP questionnaire consists of the first four questions and is considered high risk for OSA and a "yes" response to fewer questions is given with at least two "yes" answers. The STOP-Bang questionnaire was evaluated by two different methods. If at least three of the questions were given a "yes" answer at the first assessment, a high risk for OSA and a "yes" response to lesser questions were considered low risk for OSA. The second assessment was made by the University Health Network in 2014 and used low intermediate-high risk scoring criteria for OSA with various criteria. These criteria are given in Table 1.

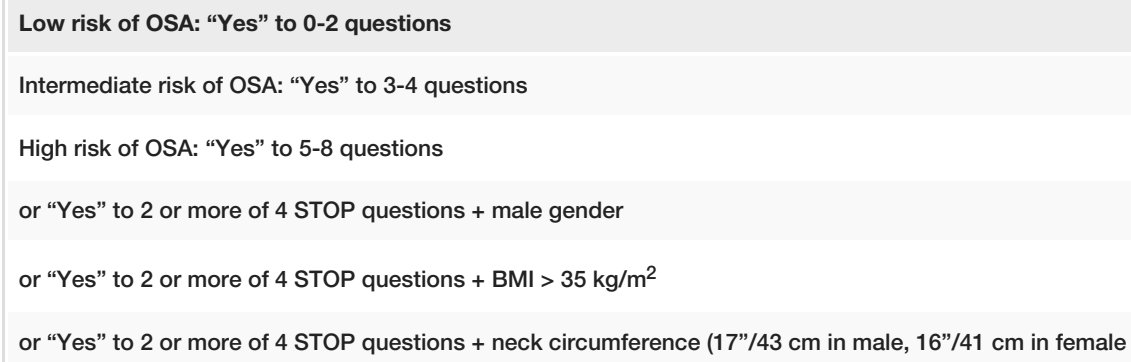

\section{TABLE 1: Updated STOP-Bang Questionnaire Scoring Criteria}

\section{Polysomnography}

In sleep laboratory, sleep structure (electroencephalogram, electrooculogram and submental electromyogram), breathing (thoracic and abdominal measurements), limb movements, nasal airflow and snoring severity were recorded. Percutaneous oxygen saturation (SpO2) and transcutaneous pulse oximetry were used for heart rate measurement. Based on the criteria of the American Academy of Sleep Medicine, the apnea-hypopnea index (AHI) value was used to determine the severity of OSA. Apnea was defined as a persistent airflow of at least 10 seconds. Periods in which airflow decreased by more than $30 \%$ and $\mathrm{SpO} 2$ decreased by more than $4 \%$ for at least 10 seconds were defined as hypopnea. The total number of apnea and hypopnea events per hour was also calculated as AHI. Thus, if the AHI value is $\leqslant 5$, the patient was considered not to have OSA. The AHI score was defined as mild OSA of 5-15, moderate OSA of 15-30, and severe OSA of $>30$.

\section{Statistical analysis}

The data was saved by creating a Microsoft Excel file. Analyses of the data were made with the program "SPSS for Windows, Version 22.0" (IBM Corp., Armonk, NY). Descriptive statistics, percent for nominal variables, mean \pm standard deviation or interquartile range for continuous variables are used. The questions of demographic data and STOP-Bang questionnaire were first divided into three groups according to obesity classification and then divided into risk groups according to three different questionnaire scores and evaluated by Pearson analysis method. The predictive parameters (sensitivity, specificity, and positive and negative predictive values) for alternative scoring models in obese patients were analyzed. Area under the curve for each score was STOP-Bang questionnaire calculated. A p-value of $<0.05$ was considered for statistical significance in the evaluations.

\section{Results}




\section{Cureus}

A total of 275 obese patients referred to the Working Sleep Disease Polyclinic were included. However, 217 patients were considered eligible for the study. Gender, age, BMI, neck circumference, cigarette and alcohol use, and additional diseases are included in Table 2 according to obesity classification. According to the classification made in the study, there was a significant relationship between neck circumference and BMI $(\mathrm{p}<0.05)$. In addition, there is a significant relationship between diabetes, asthma and hypothyroidism and BMI in patients who have additional diseases $(\mathrm{p}<0.05)$.

\begin{tabular}{|c|c|c|c|c|}
\hline & Obesity Class I & Obesity Class II & Obesity Class III (Morbidly) & Total \\
\hline $\mathrm{n}$ & 104 & 66 & 47 & 217 \\
\hline Gender, n (F/M) & $21 / 83$ & $19 / 47$ & $19 / 28$ & 59/158 \\
\hline Age, years & $50.5 \pm 11$ & $50.3 \pm 9$ & $49.7 \pm 11$ & $50.2 \pm 10$ \\
\hline BMI, kg/m² & $32 \pm 1$ & $36.8 \pm 1$ & $43.4 \pm 2$ & $35.9 \pm 4$ \\
\hline Neck, cm & $42.5 \pm 3$ & $43.1 \pm 3$ & $44.4 \pm 3$ & $43.1 \pm 3$ \\
\hline Smoke, n (\%) & $39(37.5)$ & $23(34.8)$ & $13(27.7)$ & $75(34.6)$ \\
\hline Alcohol, n (\%) & $21(20,2)$ & $9(13.6)$ & $3(6.4)$ & $33(15.2)$ \\
\hline \multicolumn{5}{|l|}{ Comorbidities } \\
\hline CAD, n (\%) & $15(14.4)$ & $14(21.2)$ & $9(19.1)$ & $38(17.5)$ \\
\hline CHF, n (\%) & $2(1.9)$ & $1(1.5)$ & $3(6.4)$ & $6(2.8)$ \\
\hline Arrhythmia, n (\%) & $11(10.6)$ & $4(6.1)$ & $4(8.5)$ & $19(8.8)$ \\
\hline Hypertension, $\mathrm{n}(\%)$ & $41(39.4)$ & $35(53)$ & $25(53.2)$ & 101 (46.5) \\
\hline Diabetes, n (\%) & $19(18.3)$ & $26(39.4)$ & $24(51.1)$ & $69(31.8)$ \\
\hline Asthma, $n(\%)$ & $9(8.7)$ & $10(15.2)$ & $12(25.5)$ & 31 (14.3) \\
\hline COPD, n (\%) & $9(8.7)$ & $9(13.6)$ & $6(12.8)$ & $24(11.1)$ \\
\hline Hypothyroidism, n (\%) & $7(6.7)$ & $4(6.1)$ & $9(19.1)$ & $20(9.2)$ \\
\hline
\end{tabular}

\section{TABLE 2: Demographic and anthropometric data of the obese patients}

Data are depicted as number (percentage), mean $\pm \mathrm{SD}$, or median (interquartile range).

CAD: Coronary artery disease; CHF: Congestive heart failure; COPD: Chronic obstructive pulmonary disease.

According to the obesity classification of patients, AHI, severity of OSA and alternative scoring models of STOP-Bang are shown in Table 3 . There was no statistically significant relationship between BMI, AHI, OSA and all three alternative scoring models of the patients $(p>0.05)$. 


\section{Cureus}

\begin{tabular}{|c|c|c|c|c|}
\hline & Obesity Class I & Obesity Class II & Obesity Class III (Morbidly) & Total \\
\hline AHI median (25-75th) & $39.6(21-61)$ & $48.1(24-64)$ & $53.3(23.4-78.5)$ & $45.6(22-64)$ \\
\hline \multicolumn{5}{|l|}{ Severity of OSA, $n(\%)$} \\
\hline No OSA & $2(1.9)$ & $0(0)$ & $1(2.1)$ & $3(1.4)$ \\
\hline Mild OSA & $16(15.4)$ & $7(10.6)$ & $2(4.3)$ & $25(11.5)$ \\
\hline Moderate OSA & $20(19.2)$ & $16(24.2)$ & $9(19.1)$ & $45(20.7)$ \\
\hline Severe OSA & 66 (63.5) & $43(65.2)$ & $35(74.5)$ & $144(66.4)$ \\
\hline
\end{tabular}

\section{TABLE 3: Polysomnography results: AHI, OSA severity}

AHI: Apnea-hypopnea index; OSA: Obstructive sleep apnea.

According to AHI $>5$ cut-off, gender $(p<0.001)$, age $(p=0.041)$, coronary artery disease $(p=0.001)$, congestive heart failure $(\mathrm{p}<0.001)$, arrhythmia $(\mathrm{p}<0.001)$, hypertension $(\mathrm{p}<0.001)$, hypothyroidism $(\mathrm{p}<$ $0.001)$, asthma ( $<<0.001)$, chronic obstructive pulmonary disease $(\mathrm{p}<0.001)$, STOP $(\mathrm{p}<0.001)$, STOP-BANG $(\mathrm{p}<0.001)$, modified STOP-BANG $(\mathrm{p}<0.001)$ scores were found statistically significant.

The STOP questionnaire revealed a sensitivity of $87.9 \%$ and a positive predictive value of $99.5 \%$ for patients with all OSA (p: 0.005). The STOP-Bang scoring model revealed a sensitivity of $95.3 \%$ and a positive predictive value of $99.5 \%$ for patients with all OSA $(\mathrm{p}<0.001)$. The modified STOP-Bang scoring revealed a sensitivity of $95.8 \%$ and a positive predictive value of $99.5 \%$ for patients with all OSA ( $p<0.001)$. Area under the curve of the STOP-Bang for identifying mild, moderate and severe OSA was $0.581,0.652$, and 0.675 , respectively. Whereas according to the STOP-Bang, all morbid obese patients (obesity class III, n: 47) were at high risk of OSA. In 217 obese patients, a STOP score cutoff of 3 and a STOP-Bang score cutoff of 4 provides a better balance of sensitivity and specificity for all OSA (AHI $\geqslant 5$ ). In addition, the predictive parameters between the questions in the questionnaire and the OSA diagnoses are listed in Table 4 . Table 5 shows the predictive parameters according to the OSA severity. 


\section{Cureus}

Positive response of STOP-Bang, $n$ (\%)

Q1 (Snoring)

Q2 (Tired or sleepy)

Q3 (Observed apnea)

Q4 (High blood pressure)

Score BMI $\left(>35 \mathrm{~kg} / \mathrm{m}^{2}\right)$

Score Age ( $>50$ years)

Score Neck $(>40 \mathrm{~cm})$

Score Gender

Alternative scoring models of STOP-Bang questionnaire

STOP, n (LR-HR)

STOP-Bang, n (LR-HR)

STOP-Bang modifying, $n$ (LR-IR-HR)
Obesity Class I

Obesity Class II

Obesity Class III (Morbidly)

44 (93.6)

$40(85.1)$

$41(87.2)$

24 (51.1)

47 (100)

20 (42.6)

36 (76.6)

28 (59.6)

47 (71.2)

83 (79.8)

$(50)$

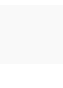

15-89

9-95

7-59

$6-41$

0-47

0-3-44
Total

204 (94)

192 (88.5)

193 (88.9)

109 (50.2)

99 (45.6)

106 (48.8)

115 (53)

158 (72.8)

28-189

$12-205$

3-8-206

\section{TABLE 4: STOP-Bang questionnaire data of the obese patients}

Data are depicted as number (percentage), mean $\pm \mathrm{SD}$, or median (interquartile range).

LR: Low risk; IR: Intermediate risk; HR: High risk; STOP: Snoring, tiredness, observed apnea; STOP-Bang: Snoring, tiredness, observed apnea, blood pressure, body mass index, age, neck circumference and gender. 


\section{Cureus}

\begin{tabular}{|c|c|c|c|c|}
\hline All OSA (AHI > 5) & Sensitivity (\%) & Specificity (\%) & PPV (\%) & NPV (\%) \\
\hline Q1 (Snoring) & $94.4 \%$ & $33.3 \%$ & $99.0 \%$ & $7.7 \%$ \\
\hline Q2 (Tired or sleepy) & $88.3 \%$ & $0.0 \%$ & $98.4 \%$ & $0.0 \%$ \\
\hline Q3 (Observed apnea) & $89.7 \%$ & $66.7 \%$ & $99.5 \%$ & $8.3 \%$ \\
\hline Q4 (High blood pressure) & $50.5 \%$ & $66.7 \%$ & $99.1 \%$ & $1.9 \%$ \\
\hline Score BMI (>35 kg/m²) & $45.8 \%$ & $66.7 \%$ & $99.0 \%$ & $1.7 \%$ \\
\hline Score Age (>50 years) & $49.1 \%$ & $66.7 \%$ & $99.1 \%$ & $1.8 \%$ \\
\hline Score Neck $(>40 \mathrm{~cm})$ & $53.7 \%$ & $100.0 \%$ & $100.0 \%$ & $2.9 \%$ \\
\hline Score Gender & $73.8 \%$ & $100.0 \%$ & $100.0 \%$ & $5.1 \%$ \\
\hline \multicolumn{5}{|c|}{ Moderate/Severe OSA $(\mathrm{AHI}>$ > 15) } \\
\hline Q1 (Snoring) & $95.2 \%$ & $14.3 \%$ & $88.2 \%$ & $30.8 \%$ \\
\hline Q2 (Tired or sleepy) & $87.3 \%$ & $3.6 \%$ & $85.9 \%$ & $4.0 \%$ \\
\hline Q3 (Observed apnea) & $91.5 \%$ & $28.6 \%$ & $89.6 \%$ & $33.3 \%$ \\
\hline Q4 (High blood pressure) & $50.8 \%$ & $53.6 \%$ & $88.1 \%$ & $13.9 \%$ \\
\hline Score BMI (>35 kg/m²) & $49.2 \%$ & $78.6 \%$ & $93.9 \%$ & $18.6 \%$ \\
\hline Score Age ( $>50$ years) & $49.2 \%$ & $53.6 \%$ & $87.7 \%$ & $13.5 \%$ \\
\hline Score Neck $(>40 \mathrm{~cm})$ & $54.0 \%$ & $53.6 \%$ & $88.7 \%$ & $14.7 \%$ \\
\hline Score Gender & $73.5 \%$ & $32.1 \%$ & $88.0 \%$ & $15.3 \%$ \\
\hline \multicolumn{5}{|l|}{ Severe OSA (AHI > 30) } \\
\hline Q1 (Snoring) & $96.5 \%$ & $11.0 \%$ & $68.1 \%$ & $61.5 \%$ \\
\hline Q2 (IIred or sleepy) & $84.0 \%$ & $2.7 \%$ & $63.0 \%$ & $8.0 \%$ \\
\hline Q3 (Observed apnea) & $93.1 \%$ & $19.2 \%$ & $69.4 \%$ & $58.3 \%$ \\
\hline Q4 (High blood pressure) & $50.7 \%$ & $50.7 \%$ & $67.0 \%$ & $34.3 \%$ \\
\hline Score BMI (>35 kg/m²) & $50.7 \%$ & $64.4 \%$ & $73.7 \%$ & $39.8 \%$ \\
\hline Score Age (>50 years) & $50.0 \%$ & $53.4 \%$ & $67.9 \%$ & $35.1 \%$ \\
\hline Score Neck (>40 cm) & $56.3 \%$ & $53.4 \%$ & $70.4 \%$ & $38.2 \%$ \\
\hline Score Gender & $.8 \%$ & 37.0 & $70.9 \%$ & $\%$ \\
\hline
\end{tabular}

\section{TABLE 5: Predictive parameters}

PPV: Positive predictive value; NPV: Negative predictive value; OSA: Obstructive sleep apnea.

\section{Discussion}

In the study, $\mathrm{BMI}$, neck circumference, hypertension, diabetes, mean $\mathrm{O}_{2}$ saturation, $\mathrm{BMI}$ score, neck score and gender scores were found statistically significant among obesity classes like in the study of Chung et al.. Stop-Bang scores were found statistically significant with hypertensive patients, absence of OSA, severe OSA, AHI at greater than 5, 15 and 30, Q1 (snoring), Q3 (observed apnea), Q4 (high blood pressure), BMI score, neck score. The STOP-Bang questionnaire was associated with high sensitivity and PPV at AHI $>5$ and $\mathrm{AHI}>15$ rather than $\mathrm{AHI}>30$.

Studies indicate high incidence of coronary artery disease, hypertension, cerebrovascular accidents, gastroesophageal reflux, congestive heart failure, myocardial infarction with OSA [14,15]. Hypertension was found statistically significant among high risk patients in our study too. The sensitivity varied from $76 \%$ to $96 \%$ and specificity varied from $70 \%$ to $93 \%$ [16-18]. Our sensitivity and specificity values are lower than 
those, perhaps our patients are solely obese patients. BMI, age ( $>50$ years old), neck circumference, and gender score have very high PPV for OSA (nearly 100\% at AHI > 5). Specificity was found lower as in Acar et al. study [19].

In a study, STOP-Bang questionnaire was used among surgical patients (27.5\%); in another study, $41.5 \%$ of patients found under high risk [20]. In our study, this ratio was $94.4 \%$. In a study including bariatric surgery patients, high-risk patients were $80 \%$ which is similar to our study [21].

In comparison of AHI > 5 cut-off, gender, age, hypertension were found statistically significant as in Chung et al. and Acar et al. study [20]. The STOP-Bang score cutoff of 4 provides a better balance of sensitivity and specificity for all OSA (AHI $\geqslant 5$ ), it is similar with the Chung et al.'s study [20,22].

In a recent study where OSA was evaluated among pregnant women with obesity during the second trimester of pregnancy, predictability of OSA is increased with high scores in STOP-Bang scores (score $<3:<10 \%$, score 6: $<3-68 \%$ ). Best fitted model of STOP-Bang is the model followed with Q1 (snoring). Predictability was $5 \%$ without snoring and $26.5 \%$ with snoring. In our trial also, STOP-Bang score was found significant with Q1 (snoring). In that study, hypertension prevalence was found low despite other studies reported hypertension $8-50 \%$. In our study, hypertension was found statistically significant at AHI > 5 cut-off with gender and age [23].

In a new study, STOP-Bang and polysomnography were performed among 541 Koreans where the usefulness of the items constituting is explored: SOPBAG (when T and A, tiredness and age, are excluded) had a better area under the curve (AUC) of 0.811 , sensitivity $89.1 \%$ and specificity $57.4 \%$ than AUC of 0.809 , sensitivity $71.7 \%$ and specificity $77.9 \%$ with STOP-Bang for Koreans. But the statistical significance between two AUC values needs to be further evaluated. This study's AUCs are better than our study's AUCs defining mild, moderate and severe OSA as $0.581,0.652$, and 0.675 , respectively [24].

In a study fulfilled among hemodialysis patients, sleep-disordered breathing (SDB) is very common among dialysis patients (70\%). STOPBANG-fixed $89 \%$ of patients had SDB. Polysomnography-fixed $86 \%$ had obstructive sleep apnoea and median AHI was 34.5/h. Oximetry and STOPBANG scores were positively correlated $(\mathrm{r}=0.62, \mathrm{p}=0.0001 ; \mathrm{r}=0.48, \mathrm{p}<0.0001)$. Neck circumference (OR: $1.20 ; 95 \%$ CI: $1.07-1.34 ; \mathrm{p}=$ 0.02 ) and haemoglobin (OR: 0.93 ; 95\% CI: 0.88-0.97; $\mathrm{p}=0.003$ ) were found independently associated with SDB. In our study too, neck scores found statistically significant with STOPBANG scores [25].

\section{Conclusions}

The STOP-Bang and STOP questionnaires are found as a valid scale among Turkish obese OSA patients in this study. The STOP-Bang scoring model revealed a sensitivity of $95.3 \%$ and a positive predictive value of 99.5\%. The STOP questionnaire revealed a sensitivity of $87.9 \%$ and a positive predictive value of $99.5 \%$. The modified STOP-Bang scoring revealed a sensitivity of $95.8 \%$ and a positive predictive value of $99.5 \%$, for patients with all OSA. These questionnaires are useful and cost-effective tools that can be applied to all OSA patients rather than polysomnography and oxymetry. There are very few studies performed among obese patients, this study is one of them. Our limitation was that we had fewer number of patients with Obesity Class III $(n=47)$ rather than Obesity Class I and II patients. Higher number of obese OSA patients could reveal better results.

\section{Additional Information \\ Disclosures}

Human subjects: Consent was obtained by all participants in this study. University of Health Sciences Health Practice \& Research Center issued approval Meeting No: 4, Date: 03 May 2017, Decision No: 2. "The Predictive Performance of the STOP-Bang Questionnaire in Obstructive Sleep Apnea Screening of Obese Population at Sleep Clinical Setting" study which is planned to be achieved by our hospital's Family Medicine Resident Dr. Burak ALTINDAG as an individual study is questioned and found suitable unanimously performed under the responsibility of the Family Medicine Training Officer, Assoc. Prof. Dr. Haluk MERGEN. Animal subjects: All authors have confirmed that this study did not involve animal subjects or tissue. Conflicts of interest: In compliance with the ICMJE uniform disclosure form, all authors declare the following: Payment/services info: All authors have declared that no financial support was received from any organization for the submitted work. Financial relationships: All authors have declared that they have no financial relationships at present or within the previous three years with any organizations that might have an interest in the submitted work. Other relationships: All authors have declared that there are no other relationships or activities that could appear to have influenced the submitted work.

\section{Acknowledgements}

This study is already presented in the "9th IPCRG Conference \& 1st Ibero-American Primary Care Respiratory Meeting, Porto, Portugal, 31 May - 2 June 2018" by same group of authors. 


\section{References}

1. Young T, Palta M, Dempsey J, Skatrud J, Weber S, Badr S: The occurrence of sleep-disordered breathing among middle-aged adults. N Engl J Med. 1993, 328:1230-1235. 10.1056/NEJM199304293281704

2. Bixler EO, Vgontzas AN, Lin HM, Ten Have T, Rein J, Vela-Bueno A, Kales A: Prevalence of sleep-disordered breathing in women: effects of gender. Am J Respir Crit Care Med. 2001, 163:608-613. 10.1164/ajrccm.163.3.9911064

3. Ip MS, Lam B, Lauder IJ, Tsang KW, Chung KF, Mok YW, Lam WK: A community study of sleep-disordered breathing in middle-aged Chinese men in Hong Kong. Chest. 2001, 119:62-69. 10.1378/chest.119.1.62

4. Ip MS, Lam B, Tang LC, Lauder IJ, Ip TY, Lam WK: A community study of sleep-disordered breathing in middle-aged Chinese women in Hong Kong: prevalence and gender differences. Chest. 2004, 125:127-134. 10.1378/chest.125.1.127

5. Kim J, In K, Kim J, et al.: Prevalence of sleep-disordered breathing in middle-aged Korean men and women . Am J Respir Crit Care Med. 2004, 170:1108-1113. 10.1164/rccm.200404-5190C

6. Udwadia ZF, Doshi AV, Lonkar SG, Singh CI: Prevalence of sleep-disordered breathing and sleep apnea in middle-aged urban Indian men. Am J Respir Crit Care Med. 2004, 169:168-173. 10.1164/rccm.200302-265OC

7. Malow BA: Approach to the patient with disordered sleep. Principles and Practice of Sleep Medicine. Kryger MH, Roth T, Dement WC (ed): WB Saunders, Philadelphia; 2005. 589-593. 10.1016/C2012-0-03543-0

8. Vaughn BV, D’Cruz OF: Cardinal manifestations of sleep disorders. Principles and Practice of Sleep Medicine. Kryger MH, Roth T, Dement WC (ed): WB Saunders, Philadelphia; 2005. 594-601. 10.1016/C20120-03543-0

9. Anch MA: The science of sleep. Sleep: A Scientific Perspective. Prentice Hall, New Jersey; 1988. 1:21.

10. Young T, Evans L, Finn L, Palta M: Estimation of the clinically diagnosed proportion of sleep apnea syndrome in middle-aged men and women. Sleep. 1997, 20:705-706. 10.1093/sleep/20.9.705

11. American Academy of Sleep Medicine: International Classification of Sleep Disorders: Diagnostic and Coding Manual (ICSD-2). American Academy of Sleep Medicine, Westchester, IL; 2005.

12. Caples SM, Gami AS, Somers VK: Obstructive sleep apnea. Ann Intern Med. 2005, 142:187-197. 10.7326/0003-4819-142-3-200502010-00010

13. Kwan SYL, Fleetham JA, Enarson DA, Chan-Yeung M: Snoring, obesity, smoking and systemic hypertension in a working population in British Columbia. Am Rev Respir Dis. 1991, 143:380.

14. Dincer FF, O'Neil W: Deleterious effects of sleep-disordered breathing on the heart and vascular system . Respiration. 2006, 73:124-130. 10.1159/000089814

15. Ing AJ, Ngu MC, Breslin AB: Obstructive sleep apnea and gastroesophageal reflux. Am J Med. 2000, 108:120125. 10.1016/s0002-9343(99)00350-2

16. Crocker BD, Olson LG, Saunders NA, Hesley MJ, McKeon JL, Allen KM, Gyulay SG: Estimation of the probability of disturbed breathing during sleep before a sleep study. Am Rev Respir Dis. 1990, 142:14-18. 10.1164/ajrccm/142.1.14

17. Viner S, Szalai JP, Hoffstein V: Are history and physical examination a good screening test for sleep apnea? . Ann Intern Med. 1991, 115:356-359. 10.7326/0003-4819-115-5-356

18. Rowley JA, Aboussouan LS, Badr MS: The use of clinical prediction formulas in the evaluation of obstructive sleep apnea. Sleep. 2000, 23:929-938. 10.1093/sleep/23.7.929

19. Acar HV, Kaya A, Yücel F, Erdem M, Eruyar Günal S, Özgen F, Dikmen B: Validation of the STOP-Bang questionnaire: an obstructive sleep apnoea screening tool in Turkish population (Article in Turkish). Turk J Anesth Reanim. 2013, 41:115-120. 10.5152/tjar.2013.46

20. Chung F, Yegneswaran B, Liao P, et al.: STOP questionnaire: a tool to screen patients for obstructive sleep apnea. Anesthesiology. 2008, 108:812-821. 10.1097/ALN.0b013e31816d83e4

21. Ong TH, Raudha S, Fook-Chong S, Lew N, Hsu AA: Simplifying STOP-BANG: use of a simple questionnaire to screen for OSA in an Asian population. Sleep Breath. 2010, 14:371-376. 10.1007/s11325-010-0350-7

22. Chung F, Yang Y, Liao P: Predictive performance of the STOP-Bang Score for identifying obstructive sleep apnea in obese patients. Obes Surg. 2013, 23:2050-2057. 10.1007/s11695-013-1006-Z

23. Pearson F, Batterham AM, Cope S: The STOP-Bang questionnaire as a screening tool for obstructive sleep apnea in pregnancy. J Clin Sleep Med. 2019, 15:705-710. 10.5664/jcsm.7754

24. Kim KT, Cho YW: Real-world STOPBANG: how useful is STOPBANG for sleep clinics? . Sleep Breath. 2019, 23:1219-1226. 10.1007/s11325-019-01806-6

25. Chu G, Suthers B, Moore L, Paech GM, Hensley MJ, McDonald VM, Choi P: Risk factors of sleep-disordered breathing in haemodialysis patients. PLoS One. 2019, 14:e0220932. 10.1371/journal.pone.0220932 